\title{
Generalized Hermite Reduction, Creative Telescoping and Definite Integration of D-Finite Functions
}

\author{
Alin Bostan \\ Inria, France \\ alin.bostan@inria.fr
}

\author{
Frédéric Chyzak \\ Inria, France \\ frederic.chyzak@inria.fr
}

\author{
Pierre Lairez \\ Inria, France \\ pierre.lairez@inria.fr
}

\author{
Bruno Salvy* \\ Inria, France \\ bruno.salvy@inria.fr
}

\begin{abstract}
Hermite reduction is a classical algorithmic tool in symbolic integration. It is used to decompose a given rational function as a sum of a function with simple poles and the derivative of another rational function. We extend Hermite reduction to arbitrary linear differential operators instead of the pure derivative, and develop efficient algorithms for this reduction. We then apply the generalized Hermite reduction to the computation of linear operators satisfied by single definite integrals of D-finite functions of several continuous or discrete parameters. The resulting algorithm is a generalization of reduction-based methods for creative telescoping.

\section{ACM Reference Format:}

Alin Bostan, Frédéric Chyzak, Pierre Lairez, and Bruno Salvy. 2018. Generalized Hermite Reduction, Creative Telescoping and Definite Integration of D-Finite Functions. In ISSAC '18: 2018 ACM International Symposium on Symbolic and Algebraic Computation, fuly 16-19, 2018, New York, NY, USA. ACM, New York, NY, USA, 8 pages. https://doi.org/10.1145/3208976.3208992
\end{abstract}

\section{INTRODUCTION}

Ostrogradsky ${ }^{1}$ [37] and Hermite [27] showed how to decompose the indefinite integral $\int R$ of a rational function $R \in \mathbb{Q}(x)$ as $U+\int A$, where $U, A \in \mathbb{Q}(x)$, and where $A$ has only simple poles and vanishes at infinity. Their contributions consist in rational algorithms to compute $A$ and $U$, that is algorithms which do not require to manipulate the roots in $\overline{\mathbb{Q}}$ of the denominator of $R$, but merely its (squarefree) factorization. The rational function $A$ is classically called the Hermite reduction of $R$. In other words, the Hermite reduction of $R$ is a canonical form of $R$ modulo the derivatives in $\mathbb{Q}(x)$ : it depends $\mathbb{Q}$-linearly on $R$, it is equal to $R$ modulo the derivatives and it vanishes if and only if $U^{\prime}=R$ for some $U \in \mathbb{Q}(x)$.

We call generalized Hermite problem the analogous question for inhomogeneous linear differential equations of arbitrary order

$$
c_{r}(x) y^{(r)}(x)+\cdots+c_{0}(x) y(x)=R(x),
$$

where $R$ and the $c_{i}$ are rational functions in $\mathbb{K}(x)$, over some field $\mathbb{K}$ of characteristic zero. In operator notation, given $L=c_{r} \partial_{x}^{r}+\cdots+$

\footnotetext{
*Also with Univ Lyon, Inria, CNRS, ENS de Lyon, Université Claude Bernard Lyon 1, LIP UMR 5668, F-69007 Lyon, France.

${ }^{1}$ Most references in symbolic integration attribute to Ostrogradsky an algorithm to compute $U$ and $A$ based on linear algebra. As a matter of fact, Ostrogradsky introduced before Hermite a polynomial method, based on extended gcds. In passing, he invented an efficient algorithm for squarefree factorization, rediscovered by Yun $[47,48]$.
}

ACM acknowledges that this contribution was authored or co-authored by an employee, contractor or affiliate of a national government. As such, the Government retains a nonexclusive, royalty-free right to publish or reproduce this article, or to allow others to do so, for Government purposes only.

ISSAC '18, July 16-19, 2018, New York, NY, USA

(C) 2018 Association for Computing Machinery.

ACM ISBN 978-1-4503-5550-6/18/07...\$15.00

https://doi.org/10.1145/3208976.3208992 $c_{0} \in \mathbb{K}(x)\left\langle\partial_{x}\right\rangle$, the problem is to produce a rational function $[R]$ in $\mathbb{K}(x)$, that depends $\mathbb{K}$-linearly on $R$, that is equal to $R$ modulo the image $L(\mathbb{K}(x))$ and that vanishes if and only if $R$ is in $L(\mathbb{K}(x))$.

Equations like Eq. (1) occur in relation to integrating factors, and ultimately to creative telescoping. If $L^{*}$ denotes the adjoint of $L$, defined as $L^{*}=\sum_{i=0}^{r}\left(-\partial_{x}\right)^{i} c_{i}(x)$, then for any function $f$, integration by parts produces Lagrange's identity $[29, \S 5.3]$

$$
u L(f)-L^{*}(u) f=\partial_{x}\left(P_{L}(f, u)\right),
$$

where $P_{L}$ depends linearly on $f, \ldots, f^{(r-1)}, u, \ldots, u^{(r-1)}$. It follows that if $f$ is a solution of $L$, then any $R \in L^{*}(\mathbb{K}(x))$ is an integrating factor of $f$, meaning that $R f$ is a derivative of a $\mathbb{K}(x)$-linear combination of $f$ and its derivatives. The converse holds if $L$ is an operator of minimal order canceling $f$, see Proposition 4.2.

\section{Contributions}

We introduce a generalized Hermite reduction to compute such a $[R]$. Classical Hermite reduction addresses the case $L=\partial_{x}$. The algorithm operates locally at each singularity and it avoids algebraic extensions, similarly to classical Hermite reduction.

Next, we improve Chyzak's algorithm [19] for creative telescoping with the use of generalized Hermite reduction. Recall that creative telescoping is an algorithmic way to compute integrals by repeated differentiation under the integral sign and integration by parts [7]. Chyzak's algorithm repeatedly checks for the existence of a rational solution to equations like (1). A lot of time is spent checking that none exists. The use of generalized Hermite reduction makes the computation incremental and less redundant.

As a simple instance of the creative telescoping problem, let $f(t, x)$ be a function annihilated by a linear differential operator $L \in$ $\mathbb{Q}(t, x)\left\langle\partial_{x}\right\rangle$ in the differentiation with respect to $x$ only, and such that $\partial_{t}(f)=A(f)$ for another operator $A$ also in $\mathbb{Q}(t, x)\left\langle\partial_{x}\right\rangle$. We look for the minimal relation of the form

$$
\lambda_{0} f+\cdots+\lambda_{s} \partial_{t}^{s}(f)=\partial_{x}(G),
$$

with $\lambda_{0}, \ldots, \lambda_{s} \in \mathbb{Q}(t)$ and $G(t, x)$ in the function space spanned by $f$ and its derivatives, with the motive that integrating both sides with respect to $x$ may lead to something useful: on the right-hand side, the integral of the derivative simplifies, often to 0 , and on the left-hand side, the integration commutes with the $\lambda_{i} \partial_{t}^{i}$, yielding a differential equation for $\int f(t, x) \mathrm{d} x$. In Equation (3), the left-hand side is called the telescoper and the function $G(t, x)$ the certificate.

The new algorithm constructs a sequence of rational functions $R_{0}, R_{1}, \ldots$ in $\mathbb{Q}(t, x)$ such that $\partial_{t}^{i}(f)=R_{i} f+\partial_{x}(\ldots)$. Equation (3) holds if and only if $\lambda_{0} R_{0}+\cdots+\lambda_{s} R_{s}$ is an integrating factor of $f$, which in turn is equivalent to the relation

$$
\lambda_{0}\left[R_{0}\right]+\cdots+\lambda_{s}\left[R_{s}\right]=0,
$$


where [ ] is the generalized Hermite reduction with respect to $L^{*}$. Starting with $s=0$, we search for solutions of the equation above and increment $s$ until one is found. Chyzak's algorithm would solve Equation (3) at each iteration mostly from scratch, whereas the new algorithm retains the reduced forms $\left[R_{i}\right]$ from one iteration to the next, computes $\left[R_{s}\right]$ from $\left[R_{s-1}\right]$ and solves the straightforward Equation (4). This approach to creative telescoping generalizes to several parameters $t_{1}, \ldots, t_{e}$ in the integrand and to different kinds of operators acting on them, in the setting of Ore algebras.

The order of the telescopers and even the termination of the creative telescoping process are related to the confinement properties of the generalized Hermite reduction. Assuming that the poles of the rational functions $R_{0}, R_{1}, \ldots$ all lie in the same finite set, we deduce from a result of Adolphson's an upper bound on the dimension of the subspace spanned by the reductions $\left[R_{i}\right]$, which in turn bounds the order of the minimal telescoper.

\section{Previous work}

Extensions of Hermite reduction. Ostrogradsky [37] and Hermite [27] introduced a reduction for rational functions. A century later, it was extended to larger and larger classes of functions: algebraic [43], hypergeometric [3], hyperexponential [22, 26, 10,11], Fuchsian [16]. Van der Hoeven's preprint [44] considers a reduction w.r.t. the derivation operator on differential modules of finite type, so as to address the general differentially finite case. Our generalized Hermite reduction is inspired by these works. It has the same architecture as several previous ones $[10,16,11,44]$ : local reductions at finite places, followed by a reduction at infinity and the computation of an exceptional set to obtain a canonical form. Our first contribution in the present paper is to open a new direction of generalization, namely by considering reductions with respect to other operators in $\mathbb{K}(x)\left\langle\partial_{x}\right\rangle$ than the derivation operator $\partial_{x}$, acting on the space $\mathbb{K}(x)$ of rational functions. An extra benefit of our method is to avoid algebraic extensions of $\mathbb{K}$.

Index theorems. The finite-dimensionality of a function space modulo the image of a differential operator is crucial to the termination of our reduction and creative-telescoping algorithms. This finiteness, and even explicit bounds, are given by index theorems for differential equations [35]. Rational versions appeared in work by Monsky [36] related to the finiteness of de Rham cohomology, and by Adolphson [6] in a $p$-adic context, see also [41,45], and §3.6.

Creative telescoping by reduction. The use of Hermite-like reductions for computing definite integrals roots in works by Fuchs [25] and Picard $[38,39]$. In the realm of creative telescoping, this line of research forms what is called the fourth generation of creative telescoping algorithms. It was first introduced for bivariate rational functions [9], and later extended to the multivariate rational case $[12,32]$. For bivariate functions/sequences, the approach was also extended to larger classes: algebraic $[15,14]$, hyperexponential [10], hypergeometric [13,28], mixed [11], Fuchsian [16], differentially finite [44]. Our second contribution is the first reduction-based variant, for single integrals, of Chyzak's algorithm [19] for D-finite functions depending on several continuous or discrete parameters.

\section{INTRODUCTORY EXAMPLE}

\subsection{Hermite Reduction}

The equation $M(y)=a x^{2}+b x+c$, with $M$ defined by $M(y)=\left(x^{2}-1\right) y^{\prime \prime}+\left(x-2 p\left(x^{2}-1\right)\right) y^{\prime}+\left(p^{2}\left(x^{2}-1\right)-p x-n^{2}\right) y$, has a rational solution $y \in \mathbb{Q}(n, p, x)$ if and only if $a x^{2}+b x+c$ is a multiple of $p^{2} x^{2}-p x-n^{2}-p^{2}$. This follows in two steps.

First, a local analysis reveals that if $y$ has a pole at some $\alpha \in \mathbb{C}$, then so does $M(y)$ : for any $\alpha \in \mathbb{C} \backslash\{ \pm 1\}$ and for any $s>0$,

$$
\begin{aligned}
M\left((x-\alpha)^{-s}\right) & =\left(\alpha^{2}-1\right) s(s+1)(x-\alpha)^{-s-2}(1+O(x-\alpha)) \\
\text { and } M\left((x \pm 1)^{-s}\right) & = \pm s(2 s+1)(x \pm 1)^{-s-1}(1+O(x \pm 1)) .
\end{aligned}
$$

Therefore, if $M(y)$ is a polynomial then $y$ is also a polynomial.

Next, for any $s \geq 0, M\left(x^{s}\right)=p^{2} x^{s+2}+O\left(x^{s+1}\right)$, as $x \rightarrow \infty$. It follows that if $y \in \mathbb{Q}(n, p)[x]$ then $M(y) \in \mathbb{Q}(n, p)[x]$ and $\operatorname{deg}_{x} M(y)=$ $\operatorname{deg}_{x} y+2$. In particular, every polynomial of degree $\leq 2$ in $M(\mathbb{Q}(n, p, x))$ is a multiple of $M(1)=p^{2} x^{2}-p x-n^{2}-p^{2}$ over $\mathbb{Q}(n, p)$.

In $\S 3$, we define the Hermite reductions w.r.t. $M$ of $1, x$ and $x^{2}$ :

$$
[1]=1, \quad[x]=x \text {, and }\left[x^{2}\right]=\frac{x}{p}+\frac{n^{2}+p^{2}}{p^{2}},
$$

showing that $\left[p^{2} x^{2}-p x-n^{2}-p^{2}\right]=0$. Similarly, the reduction of any polynomial w.r.t. $M$ is a $\mathbb{Q}(n, p)$-linear combination of 1 and $x$.

\subsection{Creative Telescoping}

We consider the classical integral identity [40, §2.18.1, Eq. (10)]

$$
\int_{-1}^{1} \frac{e^{-p x} T_{n}(x)}{\sqrt{1-x^{2}}} \mathrm{~d} x=(-1)^{n} \pi I_{n}(p),
$$

where $T_{n}$ denotes the $n$th Chebyshev polynomial of the first kind and $I_{n}$ the $n$th modified Bessel function of the first kind. The integrand $F_{n}(p, x)$ satisfies a system of linear differential and difference equations, easily found from defining equations for $T_{n}(x)$ and $e^{-p x}$ :

$$
\begin{gathered}
\frac{\partial F_{n}}{\partial p}=-x F_{n}, \quad n F_{n+1}=\frac{\partial}{\partial x}\left(\left(x^{2}-1\right) F_{n}\right)+\left(p x^{2}+(n-1) x-p\right) F_{n}, \\
\left(1-x^{2}\right) \frac{\partial^{2} F_{n}}{\partial x^{2}}=\left(2 p x^{2}+3 x-2 p\right) \frac{\partial F_{n}}{\partial x}+\left(p^{2} x^{2}+3 p x-n^{2}-p^{2}+1\right) F_{n} .
\end{gathered}
$$

We aim at finding a similar set of linear differential-difference operators in the variables $n$ and $p$ for the integral $\int_{-1}^{1} F_{n}(p, x) \mathrm{d} x$. Note that $F_{n}$ and all its derivatives w.r.t. $x$ and $p$ and shifts w.r.t. $n$ are $\mathbb{Q}(n, p, x)$-linear combinations of $F_{n}$ and $\partial F_{n} / \partial x$.

The adjoint of the last equation is $M(y)=0$, with the operator $M$ of $\S 2.1$. The reduction w.r.t. $M$ described above makes the following computation possible. First, $F_{n}$ is not a derivative (of a $\mathbb{Q}(n, p, x)$ linear combination of $F_{n}$ and $\partial F_{n} / \partial x$ ). Indeed, $F_{n}$ is a derivative if and only if $1 \in M(\mathbb{Q}(n, p))$. Second, no $\mathbb{Q}(n, p)$-linear relation between $F_{n}$ and $\partial F_{n} / \partial p$ is a derivative, because $\partial F_{n} / \partial p=-x F_{n}$ and [1] and $[-x]$ are linearly independent over $\mathbb{Q}(n, p)$. Third, the $\mathbb{Q}(n, p)$-linear relation $p^{2}\left[x^{2}\right]+p[-x]-\left(n^{2}+p^{2}\right)[1]=0$ proves that

$$
p^{2} \frac{\partial^{2} F_{n}}{\partial p^{2}}+p \frac{\partial F_{n}}{\partial p}-\left(n^{2}+p^{2}\right) F_{n}=\frac{\partial G}{\partial x}
$$

for some $\mathbb{Q}(n, p, x)$-linear combination $G$ of $F_{n}$ and $\partial F_{n} / \partial x$. Next, the equation for $n F_{n+1}$ and the equation $\left[p x^{2}+(n-1) x-p\right]=$ 
$n x+n^{2} / p$ show that, for some $\tilde{G}$ as above,

$$
F_{n+1}+\frac{\partial F_{n}}{\partial p}-\frac{n}{p} F_{n}=\frac{\partial \tilde{G}}{\partial x}
$$

Equations (5) and (6) can then be integrated from -1 to 1 . The contour can be deformed so that the right-hand sides vanish (regardless of $G$ and $\tilde{G}$ ) and the left-hand sides provide the desired operators for the integral. These equations classically define, up to a constant factor, the function $(-1)^{n} I_{n}(p)$.

\section{GENERALIZED HERMITE REDUCTION}

Throughout this section, $M \in \mathbb{K}[x]\left\langle\partial_{x}\right\rangle$ denotes a linear differential operator with polynomial coefficients. We are interested in finding $\mathbb{K}$-linear dependency relations in $\mathbb{K}(x)$ modulo the rational image $M(\mathbb{K}(x))$ by means of a canonical form with respect to $M$.

Definition 3.1. A canonical form with respect to $M$ is a $\mathbb{K}$-linear $\operatorname{map}[]: \mathbb{K}(x) \rightarrow \mathbb{K}(x)$ such that for any $R \in \mathbb{K}(x)$ :

$$
\text { (i) }[M(R)]=0 ; \quad \text { (ii) } R-[R] \in M(\mathbb{K}(x)) \text {. }
$$

Applying [ ] to $R-[R]$ before using (ii) and (i) results in $[[R]]=[R]$.

As can be seen from Eq. (1), computing such canonical forms is tightly related to the computation of rational solutions of linear differential equations. In classical solving algorithms [2,33], bounds on the order of poles of meromorphic solutions are given by indicial equations. Next, in order to factor the computation for different inhomogeneous parts, instead of using a "universal denominator", one could at each singularity identify the polar behaviour of potential meromorphic solutions, so as to reduce rational solving to polynomial solving. This idea is what inspired the reduction algorithm for computing canonical forms in the present section ${ }^{2}$.

We begin in $\$ 3.1$ with a local analysis of $M(\mathbb{K}(x))$. Then we describe in $\$ 3.2$ a projection map $H: \mathbb{K}(x) \rightarrow \mathbb{K}(x)$ that we call weak Hermite reduction. It is not quite a canonical form. It misses an exceptional set described in $\S 3.3$, from which a canonical form is deduced. For simplicity, this is first described in the algebraic closure of the base field $\mathbb{K}$, and in $\S 3.4$ we show how to perform the computations in a rational way, i.e., without algebraic extensions.

Finally, in §3.6, we bound the dimension of the quotient $E / M(E)$, for a ring $E$ of rational functions with prescribed poles. This is relevant to getting size and complexity bounds for creative telescoping.

\subsection{Local Study}

Let $\overline{\mathbb{K}}$ be an algebraic closure of $\mathbb{K}$. For $R \in \mathbb{K}(x)$ and $\alpha \in \overline{\mathbb{K}}$, let $R_{(\alpha)}$ denote the polar part of $R$ at $\alpha$. This is the unique polynomial in $(x-\alpha)^{-1}$ with constant term zero such that $R-R_{(\alpha)}$ has no pole at $\alpha$. Similarly, the polynomial part $R_{(\infty)}$ of $R$ is the unique polynomial such that $R-R_{(\infty)}$ vanishes at infinity. By partial fraction decomposition,

$$
R=R_{(\infty)}+\sum_{\alpha \in \mathbb{K}} R_{(\alpha)}
$$

Let also ord ${ }_{\alpha} R$ denote the valuation of $R$ as a Laurent series in $x-\alpha$.

For any $\alpha \in \overline{\mathbb{K}}$, there exists a non-zero polynomial ind $\alpha \in \overline{\mathbb{K}}[s]$ and an integer $\sigma_{\alpha}$ such that for any $s \in \mathbb{Z}$,

$$
M\left((x-\alpha)^{-s}\right)=\operatorname{ind}_{\alpha}(-s)(x-\alpha)^{-s+\sigma_{\alpha}}(1+o(1)), \quad \text { as } x \rightarrow \alpha .
$$

${ }^{2}$ In the case of systems, analogues of indicial equations are more complicated; several alternatives for rational solving exist $[1,8]$, that resemble the reduction in [44].
The polynomial ind $\alpha$ is classically called the indicial polynomial of $M$ at $\alpha[46,29]$; we call the integer $\sigma_{\alpha}$ the shift of $M$ at $\alpha$. The indicial polynomial and its integer roots give a detailed understanding of the image of $M$. We similarly define the shift and the indicial polynomial at $\infty$ by the equation

$$
\begin{aligned}
& M\left(x^{s}\right)=\operatorname{ind}_{\infty}(-s) x^{s-\sigma_{\infty}}(1+o(1)), \quad \text { as } x \rightarrow \infty \\
& \text { If } M=\sum_{i=0}^{r} p_{i}(x) \partial_{x}^{i} \text {, then } \\
& \sigma_{\alpha}=\min _{0 \leq i \leq r}\left(\operatorname{ord}_{\alpha} p_{i}-i\right) \text { and } \sigma_{\infty}=\max _{0 \leq i \leq r}\left(i-\operatorname{deg} p_{i}\right) .
\end{aligned}
$$

For any $\alpha \in \overline{\mathbb{K}}$ that is not a root of the leading coefficient $p_{r}$ of $M$, we have $\operatorname{ind}_{\alpha}(s)=p_{r}(\alpha) \cdot s(s-1) \cdots(s-r+1)$ and $\sigma_{\alpha}=-r$.

\subsection{Weak Hermite Reduction}

Let im $M=M(\mathbb{K}(x))$. Let $H_{\alpha}: \overline{\mathbb{K}}(x) \rightarrow \overline{\mathbb{K}}(x)$ be the local reduction map at $\alpha$ defined by $H_{\alpha}(R)=R$ if $\operatorname{ord}_{\alpha} R \geq 0(\alpha$ is not a pole of $R)$ and by induction on $\operatorname{ord}_{\alpha} R$,

$$
H_{\alpha}(R)=\left\{\begin{array}{lr}
H_{\alpha}\left(R-\frac{c M\left((x-\alpha)^{-s-\sigma_{\alpha}}\right)}{\operatorname{ind}_{\alpha}\left(-\sigma_{\alpha}-s\right)}\right) & \text { if } \operatorname{ind}_{\alpha}\left(-\sigma_{\alpha}-s\right) \neq 0, \\
c(x-\alpha)^{-s}+H_{\alpha}\left(R-c(x-\alpha)^{-s}\right) & \text { otherwise, }
\end{array}\right.
$$

where $R=c(x-\alpha)^{-s}(1+o(1))$ as $x \rightarrow \alpha$, with $c \in \overline{\mathbb{K}} \backslash\{0\}$ and $s>0$. The induction is well-founded because in either case of the definition, the argument of $H_{\alpha}$ in the right-hand side has a valuation at $\alpha$ that is larger than $\operatorname{ord}_{\alpha} R$. By construction, we check that $R-H_{\alpha}(R) \in \operatorname{im} M$ for any $R \in \mathbb{K}(x)$.

Similarly, let $H_{\infty}: \mathbb{K}(x) \rightarrow \mathbb{K}(x)$ be the local reduction map at $\infty$ defined by $H_{\infty}(R)=R$ if $\operatorname{ord}_{\infty} R>0$ (that is $R_{(\infty)}=0$ ) and by induction on $\operatorname{ord}_{\infty}(R)$ by

$$
H_{\infty}(R)=\left\{\begin{array}{lr}
H_{\infty}\left(R-\frac{c M\left(x^{s+\sigma_{\infty}}\right)}{\operatorname{ind}_{\infty}\left(-s-\sigma_{\infty}\right)}\right) & \text { if } \operatorname{ind}_{\infty}\left(-s-\sigma_{\infty}\right) \neq 0 \\
c x^{s}+H_{\infty}\left(R-c x^{s}\right) & \text { and } s+\sigma_{\infty} \geq 0,
\end{array}\right.
$$

where $R=c x^{s}(1+o(1))$ as $x \rightarrow \infty$. By construction, we check that $R-H_{\infty}(R) \in \operatorname{im} M$ for any $R \in \mathbb{K}(x)$. The condition $s+\sigma_{\infty} \geq 0$ ensures that $M\left(x^{s+\sigma_{\infty}}\right)$ is a polynomial.

Definition 3.2. The weak Hermite reduction is the linear map $H$, seen either as $H: \mathbb{K}(x) \rightarrow \mathbb{K}(x)$ or as $H: \overline{\mathbb{K}}(x) \rightarrow \overline{\mathbb{K}}(x)$, and defined by

$$
H(R)=H_{\infty}\left(R_{(\infty)}+\sum_{\alpha \in\{\text { poles of } R\}} H_{\alpha}\left(R_{(\alpha)}\right)\right) .
$$

Proposition 3.3. For any $R \in \mathbb{K}(x)$ :

(i) $H(R)=H_{\infty} \circ H_{\alpha_{1}} \circ \cdots \circ H_{\alpha_{n}}(R)$, where $\alpha_{1}, \ldots, \alpha_{n} \in \overline{\mathbb{K}}$ are the poles of $R$;

(ii) $R-H(R) \in \operatorname{im} M$ and $H(M(R)) \in \operatorname{im} M$;

(iii) $H(H(R))=H(R)$.

Moreover:

(iv) for any $\alpha \in \overline{\mathbb{K}}$ and for any $s>0$,

$$
\operatorname{ind}_{\alpha}(s) \neq 0 \text { and } \sigma_{\alpha}-s>0 \Rightarrow H\left(M\left((x-\alpha)^{-s}\right)\right)=0 \text {; }
$$

(v) for any $s \geq 0, \operatorname{ind}_{\infty}(s) \neq 0 \Rightarrow H\left(M\left(x^{s}\right)\right)=0$.

Proof. By linearity and Equation (7), Property (i) follows from the formulas $H\left(R_{(\infty)}\right)=H_{\infty}\left(R_{(\infty)}\right)$ and $H\left(R_{(\alpha)}\right)=H_{\infty}\left(H_{\alpha}\left(R_{(\alpha)}\right)\right)$ derived from the definition of $H$. The first part of Property (ii) follows from corresponding properties for $H_{\alpha}$ and $H_{\infty}$; the second part is a consequence of applying the first to $M(R)$. 
As for the idempotence, we observe, first, that every $H(R)$ is a linear combination of some $(x-\alpha)^{-s}$, with $\operatorname{ind}_{\alpha}\left(-s-\sigma_{\alpha}\right)=0$, and $x^{s}$, with $s+\sigma_{\infty} \geq 0$ and $\operatorname{ind}_{\infty}\left(-s-\sigma_{\infty}\right)=0$; and second, that $H$ is the identity on such monomials.

As for (iv), the condition ind $\ln _{\alpha}(s) \neq 0$ together with (8) imply that $\operatorname{ord}_{\alpha} M\left((x-\alpha)^{-s}\right)=-s-\sigma_{\alpha}$, and then by definition of $H_{\alpha}$,

$$
H_{\alpha}\left(M\left((x-\alpha)^{-s}\right)\right)=H_{\alpha}\left(M\left((x-\alpha)^{-s}\right)-M\left((x-\alpha)^{-s}\right)\right)=0 .
$$

The last property is proved similarly.

\subsection{Canonical Form}

If $H$ were a canonical form, $H(M(R))$ would be 0 for any $R \in \mathbb{K}(x)$. But this property fails, and more work is required to refine $H$ into a canonical form.

Definition 3.4. The space $\operatorname{Exc}_{M}$ of exceptional functions is the $\mathbb{K}$ linear subspace of $\mathbb{K}(x)$ defined by $\operatorname{Exc}_{M}=H(\operatorname{im} M)$.

Lemma 3.5. For any $R \in \mathbb{K}(x), R \in \operatorname{im} M$ if and only if $H(R) \in \operatorname{Exc}_{M}$.

Proof. The direct implication is the definition of $\operatorname{Exc}_{M}$. For the converse, assume $H(R)=H(M(U))$ for some $U$. As $(R-M(U))-$ $H(R-M(U))=M(V)$ for some $V$ by Prop. 3.3 (ii), $R=M(U+V)$.

The generalized Hermite reduction is not a canonical form, but it is strong enough to ensure that $\operatorname{Exc}_{M}$ is finite-dimensional over $\mathbb{K}$. Proposition 3.6. Over $\overline{\mathbb{K}}$, the vector space $\operatorname{Exc}_{M}$ is generated by the finite family

(a) $H\left(M\left((x-\alpha)^{-s}\right)\right)$ with $\alpha \in \operatorname{Sing}(M), s>0$ and $\operatorname{ind}_{\alpha}(-s)=0$,

(b) $H\left(M\left((x-\alpha)^{-s}\right)\right)$ with $\alpha \in \operatorname{Sing}(M), 0<s \leq \sigma_{\alpha}$,

(c) $H\left(M\left(x^{s}\right)\right)$ with $s \geq 0$ and $\operatorname{ind}_{\infty}(-s)=0$,

where $\operatorname{Sing}(M) \subset \overline{\mathbb{K}}$ is the set of singularities of $M$ (the zeroes of its leading coefficient).

Proof. The elements $(x-\alpha)^{-s}(\alpha \in \overline{\mathbb{K}}, s>0)$ and $x^{s}(s \geq 0)$ form a basis of $\overline{\mathbb{K}}(x)$. In particular, $\operatorname{Exc}_{M}$, by definition, is generated by the $H\left((x-\alpha)^{-s}\right)$ and $H\left(x^{s}\right)$. By Proposition 3.3 (iv) and (v), $H\left(M\left((x-\alpha)^{-s}\right)\right)=0$ when $\operatorname{ind}_{\alpha}(-s) \neq 0$ and $s<\sigma_{\alpha}$. Similarly, $H\left(M\left(x^{s}\right)\right)=0$ when $\operatorname{ind}_{\infty}(-s) \neq 0$. Moreover, any $\alpha \in \overline{\mathbb{K}}$ such that $\operatorname{ind}_{\alpha}$ has a negative root or $\sigma_{\alpha}>0$ is a singularity of $M$. Therefore, the only nonzero generators of $\operatorname{Exc}_{M}$ belong to the set given in the statement.

Example 3.7. Let $M=x^{10} \partial_{x}$. We compute $\operatorname{ind}_{\alpha}(s)=-\alpha^{10} s$ for any $\alpha \in \overline{\mathbb{K}}$ and $\operatorname{ind}_{\infty}(s)=s$. Moreover $\sigma_{\alpha}=-1$ for $\alpha \notin\{0, \infty\}$, $\sigma_{0}=9$ and $\sigma_{\infty}=-9$. It follows that

$\operatorname{Exc}_{M}=\operatorname{Vect}\left\{H\left(M\left(x^{-9}\right)\right), \ldots, H\left(M\left(x^{-1}\right)\right)\right\}=\operatorname{Vect}\left\{1, x, \ldots, x^{8}\right\}$. Lemma 3.8. Given a finite-dimensional $\mathbb{K}$-linear subspace $W \subset \mathbb{K}(x)$, there is a unique idempotent linear map $\rho_{W}: \mathbb{K}(x) \rightarrow \mathbb{K}(x)$ such that: (i) $W=\operatorname{ker} \rho_{W}$; (ii) for any $R \in \mathbb{K}(x)$, the degree of the numerator of $\rho_{W}(R)$ is minimal among all $S \in \mathbb{K}(x)$ with $R-S \in W$.

The following proof gives an algorithm for computing $\rho_{W}$.

Proof. When $W \subset \mathbb{K}[x]$, the value $\rho_{W}(R)$ is the result of Gaussian elimination applied in the monomial basis to the polynomial part of $R$ with the elements of $W$.

In the general case, we write $W=Q^{-1} V$, for some subspace $V \subset$ $\mathbb{K}[x]$ and $Q \in \mathbb{K}[x]$, and define $\rho_{W}(R)=Q^{-1} \rho_{V}(Q R)$. The two properties are easily checked.

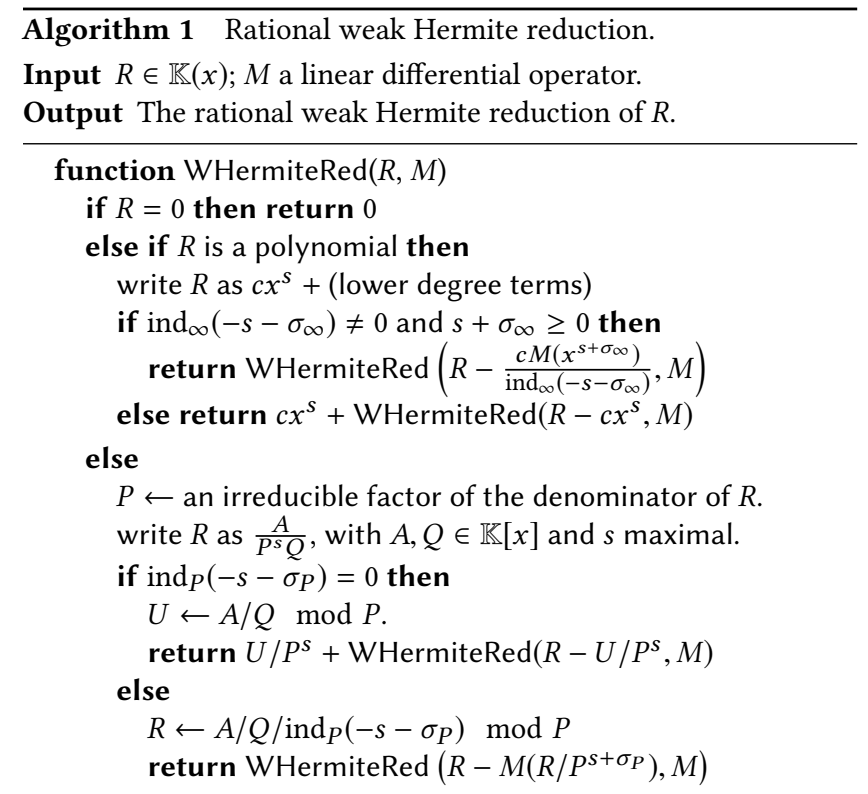

Definition 3.9. The generalized Hermite reduction with respect to $M$ is the map []$: \mathbb{K}(x) \rightarrow \mathbb{K}(x)$ defined by $[R]=\rho_{\operatorname{Exc}_{M}}(H(R))$.

THeOREM 3.10. The map [] is a canonical form with respect to $M$.

Proof. We check the properties of Definition 3.1. Let $R \in \mathbb{K}(x)$. First, $[M(R)]=0$ because $H(M(R)) \in \operatorname{Exc}_{M}$ (Lemma 3.5) and then $\rho_{\operatorname{Exc}_{M}}(H(M(R)))=0$, by Proposition 3.3 (ii) and the construction of $\rho_{\operatorname{Exc}_{M}}$. Second, $R-[R] \in \operatorname{im} M$ because $R-H(R) \in \operatorname{im} M$ (Proposition 3.3) and $H(R)-\rho_{\operatorname{Exc}_{M}}(H(R)) \in \operatorname{Exc}_{M} \subset \operatorname{im} M$.

\subsection{Rational Generalized Hermite Reduction}

In most cases, computing Hermite reduction as it is defined above would require to work with algebraic extensions of the base field. If $P \in \mathbb{K}[x]$ is a monic irreducible polynomial and $\alpha$ a root of $P$, the reduction can be performed simultaneously at all roots of $P$ without introducing algebraic extensions.

The indicial equation is obtained by considering the leading coefficient of the $P$-adic expansion of $M\left(P^{-s}\right)$, see [46, §4.1, p. 107]. More precisely, there is a unique polynomial ind $P(s)$ with coefficients in $\mathbb{K}[x] /(P)$ and a unique integer $\sigma_{P}$ such that for any $s>0$,

$$
M\left(P^{-s}\right)=\operatorname{ind}_{P}(-s) P^{-s+\sigma_{P}}+O\left(P^{-s+\sigma_{P}+1}\right),
$$

as $P$-adic expansions. Since $P$ is irreducible, $\operatorname{ind}_{P}(s)$, for a given $s$, is either 0 or invertible modulo $P$. For an irreducible polynomial $P \in \mathbb{K}[x]$, and for $R=U P^{-s}+O\left(P^{-s+1}\right)$, we define

$$
H_{P}(R)=\left\{\begin{array}{lr}
U P^{-s}+H_{P}\left(R-U P^{-s}\right) & \text { if } \operatorname{ind}_{P}\left(-\sigma_{P}-s\right)=0, \\
H_{P}\left(R-M\left(U \operatorname{ind}_{\alpha}\left(-\sigma_{P}-s\right)^{-1} P^{-s-\sigma_{P}}\right)\right) & \text { otherwise, }
\end{array}\right.
$$

where $\operatorname{ind}_{\alpha}\left(-\sigma_{P}-s\right)^{-1}$ is computed $\bmod P$. This is the part of our reduction which most closely resembles the original Hermite reduction, with successive coefficients obtained by modular inversions.

Definition 3.11. The rational weak Hermite reduction is the linear map $H_{\text {rat }}: \mathbb{K}(x) \rightarrow \mathbb{K}(x)$, defined by

$$
H_{\text {rat }}(R)=H_{\infty}\left(R_{(\infty)}+\sum_{P} H_{P}\left(R_{(P)}\right)\right),
$$


where the summation runs over the irreducible factors of the denominator of $R$ and $R_{(P)} \in \mathbb{K}\left[x, P^{-1}\right]$ denotes the polar part of the $P$-adic expansion of $R$.

The maps $H$ and $H_{\text {rat }}$ satisfy the same properties, mutatis mutandis. In particular, the latter can be used to compute a canonical form in the same way as $H$. Yet, both reductions are not equal (see also §3.5.1). For example, over $\mathbb{Q}$ with $M=\left(x^{2}+1\right) \partial_{x}+10 x$, $R=\left(x^{2}+1\right)^{-5}$ and $i^{2}+1=0$,

$$
H(R)=\frac{i}{32}\left((x+i)^{-5}-(x-i)^{-5}\right) \quad \text { whereas } \quad H_{\text {rat }}(R)=R .
$$

Partial fraction decomposition and actual Hermite reduction can be performed together. This is described in Algorithm 1. Together with the algorithm for the map $\rho_{\operatorname{Exc}_{M}}$, described in the proof of Lemma 3.8, we obtain an algorithm, denoted CanonicalForm, to compute the map $\rho \circ H_{\text {rat }}$ that is a canonical form modulo $M$.

\subsection{Variants and Improvements}

3.5.1 Absolute Hermite reduction. A notion of Hermite reduction that is independent from the base field is obtained by replacing $U \cdot P^{-s}$ with $\frac{\mathrm{d}^{s-1}}{\mathrm{~d} x^{s-1}} \frac{U}{P}$ in the definition of $H_{P}$. Another benefit of this choice is that it is not necessary that $P$ is irreducible to perform the reduction, but simply that $\operatorname{ind}_{P}(s)$ is either 0 or invertible. The denominators that appear in the computation can be factored on the fly into factors with the required property: when some ind $P(s)$ is neither 0 nor invertible modulo $P$, a gcd computation gives a non-trivial divisor of $P$.

3.5.2 Reduction to the polynomial case. The hypothesis that the differential operator $M$ has polynomial coefficients is important for the correctness of Algorithm 1. To compute canonical forms modulo an operator $M$ with rational coefficients, it is sufficient to find a polynomial $Q$ such that $M Q$ has polynomial coefficients and then, to compute canonical forms modulo $M Q$ with the algorithms above. Indeed, the image of $\mathbb{K}(x)$ by $M Q$ and $M$ are the same. The smallest such $Q$ is the gcd of the denominators of the coefficients of the adjoint of $M$.

3.5.3 Rational factors. The following observation can be used to speed up the computation.

LemmA 3.12. Let $L, M \in \mathbb{K}[x]\left\langle\partial_{x}\right\rangle$ and $A, B$ in $\mathbb{K}(x)$ such that $M A=$ $B L$. If []$_{L}$ is a canonical form w.r.t. L, then []$_{M}: R \in \mathbb{K}(x) \mapsto$ $B[R / B]_{L}$ is a canonical form w.r.t. $M$.

Proof. We check the properties of Def. 3.1: $[M(y)]_{M}=B\left[L\left(A^{-1} y\right)\right]_{L}$ is 0 and $R-[R]_{M}=B\left(R / B-[R / B]_{L}\right)$ is in $B(\operatorname{im} L)=\operatorname{im} M$.

Lemma 3.12 may be used with $A=B=\prod_{\alpha}(x-\alpha)^{m_{\alpha}}$, where $m_{\alpha}$ is the smallest negative integer root of the indicial polynomial of $M$ at $\alpha$, and 0 if none exists. This is mostly useful for equations of order 1 , since the corresponding $\alpha$ is not a singularity of the new operator, which becomes smaller. The rational function $A$ plays the role of the shell in previous reduction-based algorithms $[10,11]$.

\subsection{Dimension of the Quotient with Fixed Poles}

Let $P \in \mathbb{K}[x]$ be a squarefree polynomial and let $E_{P}=\mathbb{K}\left[x, P^{-1}\right]$. Let $\operatorname{ker} M \subset \mathbb{K}(x)$ be the space of rational solutions of $M$. Let $r$ be the order of $M$ and $d$ the maximal degree of its coefficients.
Proposition 3.13 (Adolphson [6, Sec. 5, Prop. 1]).

$$
\begin{aligned}
\operatorname{dim}_{\mathbb{K}} E_{P} / M\left(E_{P}\right) & =\operatorname{dim}_{\mathbb{K}}\left(E_{P} \cap \operatorname{ker} M\right)-\sigma_{\infty}-\sum_{P(\alpha)=0} \sigma_{\alpha} \\
& \leq(\operatorname{deg} P+1) \cdot r+d .
\end{aligned}
$$

Sketch of the Proof. Let $Z=\{\alpha \in \overline{\mathbb{K}} \mid P(\alpha)=0\}$. Given $\operatorname{deg} P+$ 1 positive integers $s_{\infty}$ and $s_{\alpha}(\alpha \in Z)$, let $E_{P}(s)$ denote the subspace of all $R \in E_{P}$ such that the pole order at $\alpha$ is at most $s_{\alpha}$ for $\alpha \in$ $Z \cup\{\infty\}$, that is all elements $R \in E_{P}(s)$ of the form

$$
R=\sum_{\alpha \in Z} \sum_{s=1}^{s_{\alpha}} \frac{c_{\alpha, s}}{(x-\alpha)^{s}}+\sum_{s=0}^{s_{\infty}} c_{\infty, s} x^{s} .
$$

We choose $s_{\alpha}$ and $s_{\infty}$ large enough so that $\operatorname{ker} M \subset E_{P}(s)$. Let $t_{\alpha}=$ $s_{\alpha}-\sigma_{\alpha}(\alpha \in Z \cup\{\infty\})$. We check $M\left(E_{P}(s)\right) \subseteq E_{P}(t)$ and that a basis of $E_{P}(t) / M\left(E_{P}(s)\right)$ induces a basis of $E_{P} / M\left(E_{P}\right)$. The bounds $-\sigma_{\alpha} \leq$ $r,-\sigma_{\infty} \leq d$ and $\operatorname{dim} \operatorname{ker} M \leq r$ give the inequality.

\section{CREATIVE TELESCOPING}

The method of creative telescoping is an approach to the computation of definite sums and integrals of objects characterized by linear functional equations. The notion of linear functional equation is formalized by Ore algebras. In this part, we consider the Ore algebra $\mathbb{A}=\mathbb{K}(x)\left\langle\partial_{x}, \partial_{1}, \ldots, \partial_{e}\right\rangle$, where $\partial_{x}$ is the differentiation with respect to $x$ and $\partial_{1}, \ldots, \partial_{e}$ are arbitrary Ore operators. In the most typical case, $\mathbb{K}=\mathbb{Q}\left(t_{1}, \ldots, t_{e}\right)$ and each $\partial_{i}$ is either the differentiation with respect to $t_{i}$ or the shift $t_{i} \mapsto t_{i}+1$.

For a given function $f$ in a function space on which $\mathbb{A}$ acts, the annihilating ideal of $f$ is the left ideal ann $f \subseteq \mathbb{A}$ of all operators that annihilate $f$. For example, the annihilating ideal in $\mathbb{K}(x)\left\langle\partial_{x}\right\rangle$ of $f=\sin (x)$ is generated by $\partial_{x}^{2}+1$ because $\sin ^{\prime \prime}(x)=-\sin (x)$.

A left ideal $\mathcal{I}$ is $D$-finite if the quotient $\mathbb{A} / \mathcal{I}$ is a finite-dimensional vector space over $\mathbb{K}(x)$. A function is called $D$-finite if its annihilating ideal is D-finite. We refer to $[18,20,21]$ for an introduction to Ore algebras, creative telescoping and their applications.

Given a D-finite function $f$, the problem of creative telescoping is the computation of a generating set of the telescoping ideal of $f$ w.r.t. $x$, or of its residue class in $\mathbb{A} / \operatorname{ann} f$. This is by definition the left ideal $\mathcal{T}_{f} \subset \mathbb{K}\left\langle\partial_{1}, \ldots, \partial_{e}\right\rangle$ of all operators $T$ such that $T+\partial_{x} G \in$ ann $f$ for some $G \in \mathbb{A}$; equivalently,

$$
\mathcal{T}_{f}=\left(\operatorname{ann} f+\partial_{x} \mathbb{A}\right) \cap \mathbb{K}\left\langle\partial_{1}, \ldots, \partial_{e}\right\rangle .
$$

Example 4.1. In $\S 2.2$, we use the Ore algebra $\mathbb{K}(x)\left\langle\partial_{x}, \partial_{1}, \partial_{2}\right\rangle$, with $\partial_{1}=d / d p$ and $\partial_{2}=S_{n}$ the shift w.r.t. $n$. The annihilating ideal $\mathcal{I}$ of $F_{n}(p, x)$ is generated by three operators, one for each functional equation. It is $D$-finite and the quotient $\mathbb{A} / \mathcal{I}$ has dimension 2 , with basis 1 and $\partial_{x}$. The telescoping ideal of $F_{n}(p, x)$ (or, equivalently, of $1 \in \mathbb{A} / \mathcal{I})$ is generated by $p^{2} \partial_{p}^{2}+p \partial_{p}-\left(n^{2}+p^{2}\right)$ and $p S_{n}+p \partial_{p}-n$.

\subsection{Cyclic Vector}

Let $\mathcal{I} \subseteq \mathbb{A}$ be a D-finite ideal and let $r$ be the dimension of $\mathbb{A} / \mathcal{I}$ over $\mathbb{K}(x)$. We denote $L(\gamma)$ the multiplication of an operator $L \in \mathbb{A}$ and a residue class $\gamma \in \mathbb{A} / \mathcal{I}$.

Let $\gamma \in \mathbb{A} / \mathcal{I}$ be a cyclic vector with respect to $\partial_{x}$. This means that $\Gamma=\left\{\gamma, \partial_{x}(\gamma), \ldots, \partial_{x}^{r-1}(\gamma)\right\}$ is a basis of $\mathbb{A} / \mathcal{I}$; or, equivalently, that every $f \in \mathbb{A} / \mathcal{I}$ can be written $A_{f}(\gamma)$ for some $A_{f} \in \mathbb{K}(x)\left\langle\partial_{x}\right\rangle$.

Let $L \in \mathbb{K}[x]\left\langle\partial_{x}\right\rangle$ be a minimal annihilating operator of $\gamma$, that is $L(\gamma)=0$ and $L$ has order $r$ (because $\Gamma$ is a basis, there is no non-zero lower order annihilating operator for $\gamma$ ). A cyclic vector 
always exists when $\mathcal{I}$ is $D$-finite [17,5]. It plays a role analogous to that of primitive elements for 0-dimensional polynomial systems.

For $1 \leq i \leq e$, we define a $\mathbb{K}$-linear map $\lambda_{i}: \mathbb{K}(x) \rightarrow \mathbb{K}(x)$ as follows. First, we can write $\partial_{i}(\gamma)=B_{i}(\gamma)$ for some operator $B_{i} \in \mathbb{K}(x)\left\langle\partial_{x}\right\rangle$. Next, let $\sigma_{i}$ and $\delta_{i}$ be the maps ${ }^{3}$ such that $\partial_{i} R=$ $\sigma_{i}(R) \partial_{i}+\delta_{i}(R)$ for any $R \in \mathbb{K}(x)$. Finally, we define for $R \in \mathbb{K}(x)$

$$
\lambda_{i}(R)=B_{i}^{*}\left(\sigma_{i}(R)\right)+\delta_{i}(R)
$$

where $B_{i}^{*}\left(\sigma_{i}(R)\right) \in \mathbb{K}(x)$ is the result of applying the adjoint operator $B_{i}^{*}$ to $\sigma_{i}(R)$, not the operator $B_{i}^{*} \sigma_{i}(R)$.

Proposition 4.2. With the notation above:

(i) $f=A_{f}^{*}(1) \gamma+\partial_{x}(Q)$, for some $Q \in \mathbb{A} / \mathcal{I}$.

Moreover, for any $R \in \mathbb{K}(x)$ :

(ii) $\partial_{i}(R \gamma)=\lambda_{i}(R) \gamma+\partial_{x}(Q)$, for some $Q \in \mathbb{A} / \mathcal{I}$.

(iii) $R \gamma \in \partial_{x}(\mathbb{A} / \mathcal{I})$ if and only if $R \in L^{*}(\mathbb{K}(x))$.

Proof. Using that $f=A_{f}(\gamma)$, Lagrange's identity (2) shows that $1 A_{f}(\gamma)-A_{f}^{*}(1) \gamma=\partial_{x}(Q)$ for some $Q$. This gives (i). Similarly, using the commutation rule for $\partial_{i}$ and the definition of $B_{i}$ yields (ii). Property (iii) is shown by Abramov and van Hoeij [4, Prop. 3].

Example 4.3 (Continuing Example 4.1). The element $1 \in \mathbb{A} / \mathcal{I}$ is a cyclic vector since $\left\{1, \partial_{x}\right\}$ is a basis of the quotient.

Actual computations are performed using a Gröbner basis of $\mathcal{I}$ and linear algebra in the finite-dimensional $\mathbb{K}(x)$-vector space $\mathbb{A} / \mathcal{I}$.

\subsection{Creative Telescoping by Reduction}

We now present our algorithm (Algorithm 2) based on generalized Hermite reduction for the computation of the telescoping ideal $\mathcal{T}_{f}$ for an element $f$ of some D-finite quotient $\mathbb{A} / \mathcal{I}$. The element $f$ is often 1, as in Example 4.1.

In the same way as Chyzak's algorithm [19], ours iterates over monomials in $\partial_{1}, \ldots, \partial_{e}$ by a strategy reminiscent of the FGLM algorithm [24]. Each iteration finds either a new generator of $\mathbb{K}\left\langle\partial_{1}, \ldots, \partial_{e}\right\rangle / \mathcal{T}_{f}$ or a new element in $\mathcal{T}_{f}$. Let [ ] be the generalized Hermite reduction with respect to $L^{*}$, the adjoint of the minimal annihilating operator of the cyclic vector $\gamma$. Since every visited monomial $\mu$ (but the first) can be written $\partial_{i} v$ for a previously visited monomial $v$, we define $F$ inductively by the formula $F_{\mu}=\left[\lambda_{i}\left(F_{v}\right)\right]$ and the base case $F_{1}=A_{f}^{*}(f)$. With Prop. 4.2 and Theorem 3.10, we check that $\mu(f)=F_{\mu} \gamma+\partial_{x}\left(Q_{\mu}\right)$, for some $Q_{\mu} \in \mathbb{A} / \mathcal{I}$ and that

$$
a_{1} \mu_{1}+\cdots+a_{s} \mu_{s} \in \mathcal{T}_{f} \quad \Leftrightarrow \quad a_{1} F_{\mu_{1}}+\cdots+a_{s} F_{\mu_{s}}=0 .
$$

THEOREM 4.4. On input $\mathcal{I}$, Algorithm 2 terminates if and only if the telescoping ideal $\mathcal{T}_{f}$ is D-finite. It outputs a Gröbner basis of $\mathcal{T}_{f}$ for the grevlex monomial ordering.

Proof. By construction, when a monomial is added to the set $R$, it is not a multiple of another monomial in $R$. By Dickson's lemma [23], this may happen only finitely many times.

The way $\mathcal{L}$ is filled ensures that when a monomial $\mu$ is visited, every smaller monomial has been visited or is a multiple of a reducible monomial. This implies, by induction, that $Q$ is the set of all non-reducible monomials that are smaller than $\mu$, when $\mu$ is visited.

${ }^{3}$ If $\partial_{i}$ is the differentiation w.r.t. $t_{i}$, then $\sigma_{i}(R)=R$ and $\delta_{i}(R)=\partial R / \partial t_{i}$. If $\partial_{i}$ is the shift $t_{i} \mapsto t_{i}+1$, then $\sigma_{i}(R)=\left.R\right|_{t_{i} \leftarrow t_{i}+1}$ and $\delta_{i}(R)=0$.

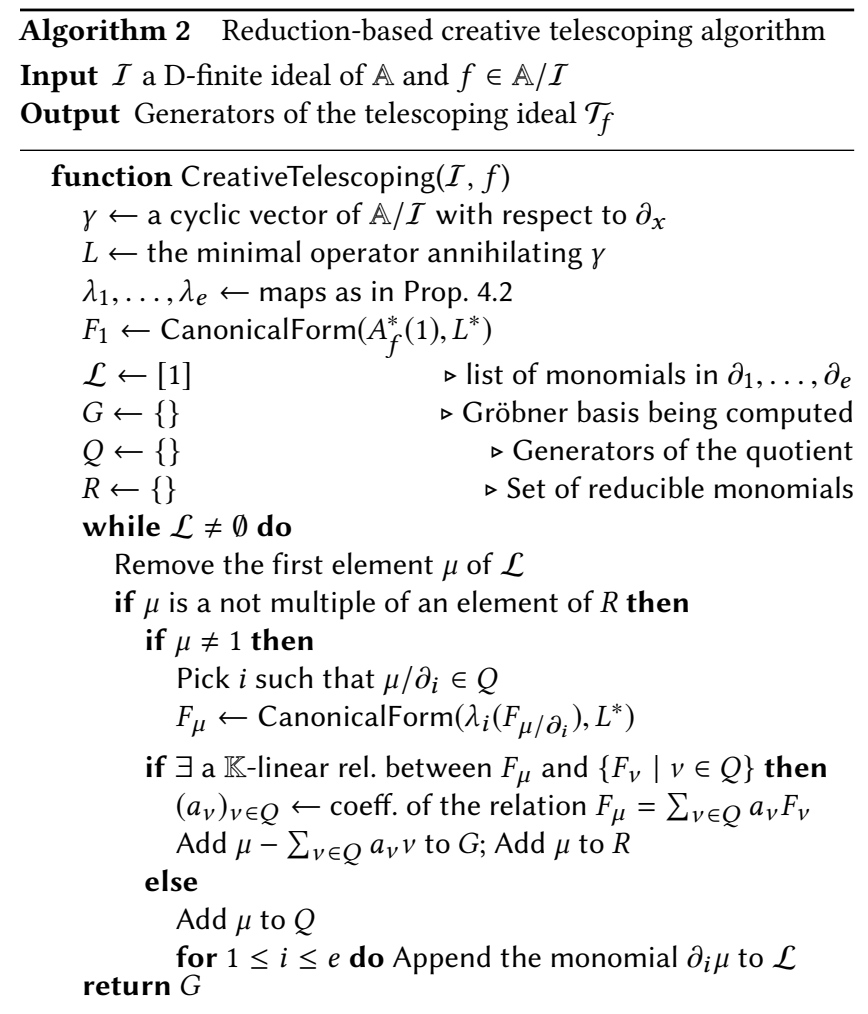

If $\mathcal{T}_{f}$ is not D-finite, then there are infinitely many non-reducible monomials and the algorithm does not terminate. Otherwise, the algorithm terminates, since neither $Q$ nor $R$ may grow indefinitely.

To check that $G$ is a Gröbner basis, we note that: $G \subset \mathcal{T}_{f}$, by the equivalence (9); the leading monomials of the elements of $G$ are the elements of $R$; and every leading monomial of an element $\mathcal{T}_{f}$ (that is a reducible monomial) is a multiple of an element of $R$.

\subsection{Variants and Improvements}

4.3.1 Different term order. As stated, Algorithm 2 computes relations by increasing total degree in $\left(\partial_{1}, \ldots, \partial_{e}\right)$. To choose a different term order, it is sufficient to change the selection of the monomial $\mu$ at the beginning of the loop and select the smallest one for the given order instead.

4.3.2 Different termination rule. Instead of waiting for the list $\mathcal{L}$ to be empty, one can stop as soon as a relation is found, and then it is the minimal one for the chosen term order. This variant does not require a D-finite ideal to terminate. Another possibility is to stop as soon as the degree of $\mu$ is larger than a predefined bound, returning all the relations that exist below this bound.

4.3.3 Certificates. While an important point of the reductionbased approaches to creative telescoping is to avoid the computation of certificates (in contrast with Chyzak's and Koutschan's algorithms that require their computation), it is also possible to modify the algorithm so that it returns a certificate for each element of the basis. Indeed, a certificate of the generalized Hermite reduction of $\S 3$ can be propagated through the algorithms. 


\subsection{D-finiteness of the Telescoping Ideal}

In the general case, the telescoping ideal $\mathcal{T}_{f}$ of a D-finite function $f$ need not be D-finite. However, when the auxiliary operators $\partial_{1}, \ldots, \partial_{e}$ are differentiation operators (as opposed to shift operators for example), then $\mathcal{T}_{f}$ is always D-finite if $f$ is; this is a well-known result in the theory of D-finiteness and holonomy [42]. We give here a new proof of this fact, as a corollary of a more general sufficient condition for general Ore operators.

Definition 4.5. A D-finite function $f$ is singular (w.r.t. $\partial_{x}$ ) at $\alpha \in \overline{\mathbb{K}}$ if every nonzero operator $L \in \mathbb{K}(x)\left\langle\partial_{x}\right\rangle$ such that $L(f)=0$ is singular at $\alpha$. The singular set (w.r.t. $\partial_{x}$ ) of $f$, denoted $\operatorname{Sing}(f)$, is the set of all singular points of $f$.

Let $\Theta=\left\{1, \partial_{1}, \partial_{2}, \ldots, \partial_{1}^{2}, \partial_{1} \partial_{2}, \ldots\right\}$ be the set of all monomials in the variables $\partial_{1}, \ldots, \partial_{e}$.

Theorem 4.6. For any D-finite function $f$, if $\cup_{\mu \in \Theta} \operatorname{Sing}(\mu(f))$ is finite, then $\mathcal{T}_{f}$ is $D$-finite.

Proof. Let $\gamma$ be a cyclic vector of $\mathbb{A} / \operatorname{ann} f$ w.r.t. $\partial_{x}$ with minimal annihilating operator $L \in \mathbb{K}(x)\left\langle\partial_{x}\right\rangle$ of order $r$. For $\mu \in \Theta$, let $A_{\mu(f)} \in \mathbb{K}(x)\left\langle\partial_{x}\right\rangle$ of order $<r$ be such that $\mu(f)=A_{\mu(f)}(\gamma)$, as in $\S 4.1$, and let $R_{\mu}=A_{\mu(f)}^{*}(1) \in \mathbb{K}(x)$, so that $\mu(f)=R_{\mu} \gamma+\partial_{x}\left(G_{\mu}\right)$, for some $G_{\mu} \in \mathbb{A} / \operatorname{ann} f$. By Proposition 4.2, the $\mathbb{K}$-linear map $\phi: \mathbb{K}\left\langle\partial_{1}, \ldots, \partial_{n}\right\rangle \rightarrow \mathbb{K}(x)$ defined by $\phi(\mu)=R_{\mu}$ induces an injective map $\mathbb{K}\left\langle\partial_{1}, \ldots, \partial_{n}\right\rangle / \mathcal{T}_{f} \rightarrow \mathbb{K}(x) /$ im $L^{*}$. The telescoping ideal $\mathcal{T}_{f}$ is D-finite if and only if the image of this map is finite-dimensional. In view of Proposition 3.13, it suffices to show that the poles of all the $R_{\mu}$ lie in a finite subset of $\overline{\mathbb{K}}$. This is obtained by proving that

$$
\bigcup_{\mu \in \Theta} \operatorname{poles}\left(R_{\mu}\right) \subseteq \operatorname{Sing}(L) \cup \bigcup_{\mu \in \Theta} \operatorname{Sing}(\mu(f)),
$$

where $\operatorname{Sing}(L)$ is the set of zeroes of the leading coefficient of $L$.

Indeed, let $\mu \in \Theta$ and $\alpha \in \overline{\mathbb{K}}$ that is not in the right-hand side. We now prove that no coefficient of $A_{\mu(f)}$ has a pole at $\alpha$, from where it follows that neither has $R_{\mu}=A_{\mu(f)}^{*}(1)$. By the hypothesis on $\alpha$, there exists $M \in \mathbb{K}(x)\left\langle\partial_{x}\right\rangle$ an annihilating operator of $\mu(f)$ regular at $\alpha$. It satisfies $M A_{\mu(f)}(\gamma)=M(\mu(f))=0$ and by minimality of $L$ it follows that $M A_{\mu(f)}=B L$ for some operator $B$. As a consequence, $0,1, \ldots, r-1$ are roots of the indicial polynomial of $M A_{\mu}(f)$. Write $A_{\mu(f)}=\sum_{i=0}^{r-1} a_{i} \partial_{x}^{i}$, for some $a_{i} \in \mathbb{K}(x)$ and let $j$ be the maximal index with ord $\operatorname{ord}_{\alpha} a_{j}=\min _{i} \operatorname{ord}_{\alpha} a_{i}$. Then $j \in\{0, \ldots, r-1\}$ and $\operatorname{ord}_{\alpha} A_{\mu(f)}\left((x-\alpha)^{j}\right)=\min _{i} \operatorname{ord}_{\alpha} a_{i}$. Then this last quantity is a zero of the indicial polynomial of $M$, which implies that it is nonnegative and thus that none of the $a_{i}$ has a pole at $\alpha$.

Recall that for $1 \leq i \leq e$, the Ore operator $\partial_{i}$ satisfies a commutation relation $\partial_{i} a=\sigma_{i}(a) \partial_{i}+\delta_{i}(a)$ for any $a \in \mathbb{K}$, where $\sigma_{i}$ is an endomorphism of $\mathbb{K}$ and $\delta_{i}$ is a $\sigma_{i}$-derivation of it. When $\partial_{i}$ is a differentiation operator, $\sigma_{i}=\mathrm{id} \mathbb{R}$.

Corollary 4.7. If $\partial_{1}, \ldots, \partial_{e}$ are differentiation operators, then $\mathcal{T}_{f}$ is $D$-finite for any $D$-finite function $f$.

Proof. It is sufficient to check that $\operatorname{Sing}(\mu(f)) \subset \operatorname{Sing}(f)$ for any monomial $\mu \in \Theta$ and then conclude by Theorem 4.6.

\begin{tabular}{lccccccc}
\hline Integral & $(11)$ & $(12)$ & $(13)$ & $(14)$ & $(15)$ & $(16)$ & $(17)$ \\
\hline redct & $13 \mathrm{~s}$ & $>1 \mathrm{~h}$ & $>1 \mathrm{~h}$ & $1.5 \mathrm{~s}$ & $1.5 \mathrm{~s}$ & $165 \mathrm{~s}$ & $53 \mathrm{~s}$ \\
HF-CT & $19 \mathrm{~s}$ & $253 \mathrm{~s}$ & $45 \mathrm{~s}$ & $232 \mathrm{~s}$ & $516 \mathrm{~s}$ & $>1 \mathrm{~h}$ & $>1 \mathrm{~h}$ \\
HF-FCT & $1.9 \mathrm{~s}^{*}$ & $2.3 \mathrm{~s}$ & $5.3 \mathrm{~s}$ & $>1 \mathrm{~h}$ & $2.3 \mathrm{~s}^{*}$ & $5.4 \mathrm{~s}$ & $2.2 \mathrm{~s}^{*}$ \\
\hline
\end{tabular}

Table 1 Comparative timings on several instances of creative telescoping. Rows are redct (new algorithm); Koutschan's HolonomicFunctions, using functions Annihilator and CreativeTelescoping (HF-CT); idem, using FindCreativeTelescoping (HF-FCT), a heuristic that does not necessarily find the minimal operators (indicated by *). All examples were run on the same machine, with the latest versions of Maple and Mathematica.

Let $M \in \mathbb{K}[x]\left\langle\partial_{x}\right\rangle$ be an annihilating operator of $g=\mu(f)$ regular at $\alpha \in \overline{\mathbb{K}} \backslash \operatorname{Sing}(f)$. The commutation rules for the differential operators imply that $\partial_{i} M=M \partial_{i}+R$, for some $R \in \mathbb{K}[x]\left\langle\partial_{x}\right\rangle$. In particular, we obtain the inhomogeneous differential equation $M\left(\partial_{i}(g)\right)=$ $R(g)$ for $\partial_{i}(g)$. Since $\alpha$ is neither a singularity of $M$ nor of $R(g)$, it follows that it is not a singularity of $\partial_{i}(g)$.

For the case of general Ore operators, we obtain with a similar proof the following result.

Corollary 4.8. For any $D$-finite function $f$, if there is a finite set $S \subset \overline{\mathbb{K}}$ such that: $(i) \sigma_{i}(S) \subseteq S$ for any $1 \leq i \leq e$ and $(i i) \operatorname{Sing}(f) \subseteq S$, then $\mathcal{T}_{f}$ is $D$-finite.

\section{EXPERIMENTS}

We present the results of a preliminary Maple implementation called redct ${ }^{4}$. Comparison is done with Koutschan's HolonomicFunctions package [31], the best available code for creative telescoping. Timings are given in Table $1^{5}$.

Koutschan's examples. Koutschan's example session [30] contains 40 integrals on which we tested our code. In most cases, our code compares well with HolonomicFunctions. There are 37 easy cases, all of whose telescopers are found in $3.5 \mathrm{sec}$. by redct, while $16 \mathrm{sec}$. are needed by HolonomicFunctions (but that includes certificates). The three other examples are (the nature of the parameters is indicated in the brackets, $C_{n}^{(\alpha)}$ denotes Gegenbauer polynomials, and $J_{1}, I_{1}$, etc. Bessel functions):

$$
\begin{gathered}
\left.\int \frac{2 J_{m+n}(2 t x) T_{m-n}(x)}{\sqrt{1-x^{2}}} \mathrm{~d} x \quad \text { [diff. } t, \text { shift } n \text { and } m\right], \\
\int_{0}^{1} C_{n}^{(\lambda)}(x) C_{m}^{(\lambda)}(x) C_{\ell}^{(\lambda)}(x)\left(1-x^{2}\right)^{\lambda-\frac{1}{2}} \mathrm{~d} x \quad[\text { shift } n, m, \ell], \\
\left.\int_{0}^{\infty} x J_{1}(a x) I_{1}(a x) Y_{0}(x) K_{0}(x) \mathrm{d} x \quad \text { [diff. } a\right] .
\end{gathered}
$$

Longer examples. We mention a few examples, some involving Gegenbauer polynomials [40, 2.21.18.2, 2.21.18.4], that take more time. The advantage of a reduction-based approach becomes visible.

$$
\begin{gathered}
\int \frac{n^{2}+x+1}{n^{2}+1}\left(\frac{(x+1)^{2}}{(x-4)(x-3)^{2}\left(x^{2}-5\right)^{3}}\right)^{n} \sqrt{x^{2}-5} e^{\frac{x^{3}+1}{x(x-3)(x-4)^{2}}} \mathrm{~d} x \quad[\text { shift } n] \\
\int C_{m}^{(\mu)}(x) C_{n}^{(v)}(x)\left(1-x^{2}\right)^{v-1 / 2} \mathrm{~d} x \quad[\text { shift } n, m, \mu, v]
\end{gathered}
$$

\footnotetext{
${ }^{4}$ Available with example sessions at https://specfun.inria.fr/chyzak/redct/.

${ }^{5}$ When our code does not terminate, time is spent computing the exceptional set. This seems to be due to apparent singularities of the operators, that become true singularities of their adjoint. Ways of circumventing this issue are under study.
} 


$$
\begin{array}{r}
\int x^{\ell} C_{m}^{(\mu)}(x) C_{n}^{(v)}(x)\left(1-x^{2}\right)^{v-1 / 2} \mathrm{~d} x \quad[\text { shift } \ell, m, n, \mu, v], \\
\int(x+a)^{\gamma+\lambda-1}(a-x)^{\beta-1} C_{m}^{(\gamma)}(x / a) C_{n}^{(\lambda)}(x / a) \mathrm{d} x \\
{[\text { diff. } a, \text { shift } n, m, \beta, \gamma, \lambda] .}
\end{array}
$$

\section{CONCLUSION}

A closer look at our algorithm reveals several aspects of the complexity of creative telescoping. To simplify the discussion, we restrict to the bivariate case and measure the arithmetic complexity, obtained by counting arithmetic operations in $\mathbb{Q}$. We look for bounds in terms of the input size (order and degree of the operators at hand).

In this setting, the complexity of computing $\mathcal{T}_{f}$ is not bounded polynomially (whatever the algorithm). Consider for instance, the integral representation of Hermite polynomials

$$
H_{n}(t)=\frac{2^{n}}{i \sqrt{\pi}} \int_{-i \infty}^{i \infty}(t+x)^{n} e^{x^{2}} \mathrm{~d} x
$$

If one computes a telescoper over $\mathbb{Q}(n, t)$, then our algorithm produces the classical differential equation $y^{\prime \prime}+2 n y=2 t y^{\prime}$. However, if $n$ is a given positive integer then the minimal telescoper is the first-order factor $H_{n}(t) \partial_{t}-H_{n}^{\prime}(t)$, with coefficients of degree $n$. Its size is exponential in the bit size of the input. Thus, no algorithm computing the minimal telescoper can run in polynomial complexity.

However, in the frequent cases like this one where the set $S$ of singularities discussed in Corollary 4.8 is bounded polynomially in terms of the size of the input, then the dimension of the quotient and therefore the order of the telescopers is bounded polynomially as a consequence of Adolphson's result (Proposition 3.13). The nonpolynomial cost of minimality thus resides only in the degree of the coefficients. Note that in the differential case, polynomial time computation of non-minimal telescopers is also achieved by wellknown methods in holonomy theory, e.g., [34, proof of Lemma 3].

In our algorithm, the non-polynomial complexity arises first in the computation of the exceptional set $\operatorname{Exc}_{M}$ and next in the reductions by the elements of this set. Removing this part of the computation and using the weak Hermite reduction yields a weak form of the algorithm that does not find minimal telescopers but runs in polynomial complexity, if the set $S$ has polynomial size.

Acknowledgement. This work was supported in part by FastRelax ANR-14-CE25-0018-01.

\section{REFERENCES}

[1] S. A. Abramov. EG-eliminations. F. Differ. Equations Appl., 5(4-5):393-433, 1999.

[2] S. A. Abramov and K. Y. Kvashenko. Fast algorithms for the search of the rational solutions of linear differential equations with polynomial coefficients. In ISSAC'91, pages 267-270, 1991.

[3] S. A. Abramov and M. Petkovšek. Minimal decomposition of indefinite hypergeometric sums. In ISSAC'01, pages 7-14. ACM, 2001.

[4] S. A. Abramov and M. van Hoeij. Integration of solutions of linear functional equations. Integral Transform. Spec. Funct., 8(1-2):3-12, 1999.

[5] K. Adjamagbo. Sur l'effectivité du lemme du vecteur cyclique. C. R. Acad. Sci. Paris Sér. I Math., 306(13):543-546, 1988.

[6] A. Adolphson. An index theorem for $p$-adic differential operators. Trans. Amer. Math. Soc., 216:279-293, 1976.

[7] G. Almkvist and D. Zeilberger. The method of differentiating under the integral sign. J. Symbolic Comput., 10(6):571-591, 1990.

[8] M. A. Barkatou. On rational solutions of systems of linear differential equations. f. Symbolic Comput., 28(4-5):547-567, 1999.

[9] A. Bostan, S. Chen, F. Chyzak, and Z. Li. Complexity of creative telescoping for bivariate rational functions. In ISSAC'10, pages 203-210. ACM, 2010.
[10] A. Bostan, S. Chen, F. Chyzak, Z. Li, and G. Xin. Hermite reduction and creative telescoping for hyperexponential functions. In ISSAC'13, pages 77-84. ACM, 2013.

[11] A. Bostan, L. Dumont, and B. Salvy. Efficient algorithms for mixed creative telescoping. In ISSAC'16, pages 127-134. ACM, 2016.

[12] A. Bostan, P. Lairez, and B. Salvy. Creative telescoping for rational functions using the Griffiths-Dwork method. In ISSAC'13, pages 93-100. ACM, 2013.

[13] S. Chen, H. Huang, M. Kauers, and Z. Li. A modified Abramov-Petkovšek reduction and creative telescoping for hypergeometric terms. In ISSAC'15, pages 117-124. ACM, 2015.

[14] S. Chen, M. Kauers, and C. Koutschan. Reduction-based creative telescoping for algebraic functions. In ISSAC'16, pages 175-182. ACM, 2016.

[15] S. Chen, M. Kauers, and M. F. Singer. Telescopers for rational and algebraic functions via residues. In ISSAC'12, pages 130-137. ACM, 2012.

[16] S. Chen, M. van Hoeij, M. Kauers, and C. Koutschan. Reduction-based creative telescoping for fuchsian D-finite functions. F. Symbolic Comput., 85:108-127, 2018.

[17] R. C. Churchill and J. J. Kovacic. Cyclic vectors. In Differential Algebra and Related Topics, pages 191-218. World Scientific, 2002.

[18] F. Chyzak. Fonctions holonomes en calcul formel. $\mathrm{PhD}$ Thesis, École polytechnique, 1998.

[19] F. Chyzak. An extension of Zeilberger's fast algorithm to general holonomic functions. Discrete Math., 217(1-3):115-134, 2000.

[20] F. Chyzak. The ABC of Creative Telescoping - Algorithms, Bounds, Complexity. Accreditation to supervise research (HDR), École polytechnique, Apr. 2014.

[21] F. Chyzak and B. Salvy. Non-commutative elimination in Ore algebras proves multivariate identities. 7. Symbolic Comput., 26(2):187-227, 1998.

[22] J. H. Davenport. The Risch differential equation problem. SIAM f. Comput., 15(4):903-918, 1986

[23] L. E. Dickson. Finiteness of the Odd Perfect and Primitive Abundant Numbers with $n$ Distinct Prime Factors. Amer. 7. Math., 35(4):413-422, 1913.

[24] J. C. Faugère, P. Gianni, D. Lazard, and T. Mora. Efficient computation of zerodimensional Gröbner bases by change of ordering. F. Symbolic Comput., 16(4):329344, 1993.

[25] L. Fuchs. Die Periodicitätsmoduln der hyperelliptischen Integrale als Functionen eines Parameters aufgefasst. F. Reine Angew. Math., 71:91-127, 1870.

[26] K. Geddes, H. Le, and Z. Li. Differential rational normal forms and a reduction algorithm for hyperexponential functions. In ISSAC'04, pages 183-190, 2004.

[27] C. Hermite. Sur l'intégration des fractions rationnelles. Ann. Sci. École Norm. Sup. (2), $1: 215-218,1872$.

[28] H. Huang. New bounds for hypergeometric creative telescoping. In ISSAC'16, pages 279-286. ACM, 2016.

[29] E. L. Ince. Ordinary Differential Equations. Dover Publications, New York, 1944.

[30] C. Koutschan. Examplesv11.nb. On the HolonomicFunctions web page.

[31] C. Koutschan. Advanced Applications of the Holonomic Systems Approach. $\mathrm{PhD}$ thesis, RISC-Linz, 2009.

[32] P. Lairez. Computing periods of rational integrals. Math. Comp., 85(300):1719$1752,2016$.

[33] J. Liouville. Second mémoire sur la détermination des intégrales dont la valeur est algébrique. fournal de l'École polytechnique, 14:149-193, 1833.

[34] L. Lipshitz. The diagonal of a $D$-finite power series is $D$-finite. F. Algebra, 113(2):373-378, 1988.

[35] B. Malgrange. Sur les points singuliers des équations différentielles. Enseignement Math. (2), 20:147-176, 1974.

[36] P. Monsky. Finiteness of de Rham cohomology. Amer. F. Math., 94:237-245, 1972.

[37] M. Ostrogradsky. De l'intégration des fractions rationnelles. Bull. classe phys.math. Acad. Impériale des Sciences Saint-Pétersbourg, 4:145-167, 286-300, 1845.

[38] É. Picard. Sur les intégrales doubles de fonctions rationnelles dont tous les résidus sont nuls. Bull. Sci. Math. (2), 26:143-152, 1902.

[39] É. Picard and G. Simart. Théorie des fonctions algébriques de deux variables indépendantes, volume I (1897) and II (1906). Gauthier-Villars et fils, 1897.

[40] A. P. Prudnikov, Y. A. Brychkov, and O. I. Marichev. Integrals and series. Vol. 2. Gordon \& Breach Science Publishers, NY, second edition, 1988. Special functions.

[41] M. S. Rezaoui. Indice polynomial d'une matrice d'opérateurs différentiels. C. $R$. Acad. Sci. Paris Sér. I Math., 332(6):505-508, 2001.

[42] N. Takayama. An approach to the zero recognition problem by Buchberger algorithm. Journal of Symbolic Computation, 14:265-282, 1992.

[43] B. M. Trager. Integration of Algebraic Functions. PhD Thesis, MIT, 1984.

[44] J. van der Hoeven. Constructing reductions for creative telescoping, 2017. Technical Report, HAL 01435877, http://hal.archives-ouvertes.fr/hal-01435877/.

[45] M. van der Put and M. Reversat. A local-global problem for linear differential equations. Pacific 7. Math., 238(1):171-199, 2008.

[46] M. van der Put and M. F. Singer. Galois theory of linear differential equations, volume 328 of Grundlehren der Mathematischen Wissenschaften. Springer, 2003.

[47] D. Y. Y. Yun. On square-free decompositions algorithms. In Proc. 1976 ACM Symposium on Symbolic and Algebraic Computation, pages 26-35. ACM, 1976.

[48] D. Y. Y. Yun. Fast algorithm for rational function integration. In Proc. IFIP'77 Congr., Toronto, Ont., pages 493-498. North-Holland, 1977. 